\title{
A Functional Assay to Correlate Protein Oligomerization States with Membrane Pore Formation
}

\section{SUPPLEMENTARY INFORMATION}

Radek Šachl ${ }^{1, *}$, Sabína Čujová ${ }^{1}$, Vandana Singh ${ }^{1}$, Petra Riegerová ${ }^{1}$, Peter Kapusta ${ }^{1}$, Hans- $^{-}$ Michael Müller ${ }^{2}$, Julia P. Steringer ${ }^{2}$, Martin $\operatorname{Hof}^{1}$ and Walter Nickel ${ }^{2}$

${ }^{1} J$. Heyrovský Institute of Physical Chemistry of the Czech Academy of Sciences, Dolejškova 318223 Prague, Czech Republic;

${ }^{2}$ Heidelberg University Biochemistry Center, Im Neuenheimer Feld 328, 69120 Heidelberg, Germany

*Correspondence:

Radek Šachl

radek.sachl@jh-inst.cas.cz

Table of content

Page

1. Materials and Methods

$\begin{array}{lr}\text { Protein expression and purification } & \text { SI } 2\end{array}$

$\begin{array}{lr}\text { Preparation of GUVs } & \text { SI } 2\end{array}$

$\begin{array}{ll}\text { Microscope setup } & \text { SI } 2\end{array}$

$\begin{array}{ll}\text { Determination of the readout parameters by dual (+1)-FCS } & \text { SI } 4\end{array}$

2. Supporting Results

Functional correlation of the diffusion coefficent with membrane pore formation $\quad$ SI 5

$\begin{array}{ll}\text { 3. References } & \text { SI } 6\end{array}$ 


\section{Materials and Methods}

Protein expression and purification

His-FGF2-Y81pCMF-GFP, His-FGF2-Y81pCMF-C77/95A-GFP and His-FGF2Y81pCMF-C77/95A were expressed in E. coli strains W3110Z1 or BL21 Star (DE3), respectively. For incorporation of the unnatural amino acid p-carboxylmethylphenylalanine (pCMF; custom synthesis by ENAMINE Ltd., Kiev, Ukraine), codon 81 (tyrosine) was replaced by an amber stop codon. Transformation of a strain carrying the pEVOL-pCMF plasmid resulted in expression of recombinant His-FGF2-Y81pCMF. ${ }^{1}$ All proteins were purified in three steps via Ni-NTA affinity chromatography, heparin chromatography and size exclusion chromatography using a Superdex 75 column.

\section{Preparation of GUVs}

GUVs with a plasma membrane like lipid composition consisting of 33 mol\% phosphatidylcholine (PC), 10 mol\% phosphatidylethanolamine (PE), 5 mol\% phosphatidylserine (PS), 5 mol\% phosphatidylinositol (PI), 15 mol\% sphingomyelin (SM), $30 \mathrm{~mol} \%$ cholesterol (Chol), $1 \mathrm{~mol} \%$ Biotinyl-PE (Avanti Polar Lipids) and $0.05 \mathrm{~mol} \%$ dioleolyl-PE labelled in the headgroup by Atto-633 (Atto-633-DOPE, ATTO-TEC) were generated based on electro-swelling using platinum electrodes. ${ }^{2}$ GUVs were supplemented with either $2 \mathrm{~mol} \%$ phosphatidylinositol-4,5-bisphosphate ( $\left.\mathrm{PIP}_{2}\right)$ or a DGS-NTA lipid (Avanti Polar Lipids). The dried lipid film was hydrated with a $300 \mathrm{mM}$ sucrose solution (300 mOsmol/kg). Electro-swelling was conducted at $45^{\circ} \mathrm{C}(10 \mathrm{~Hz}, 1.5 \mathrm{~V}$ for $50 \mathrm{~min}, 2 \mathrm{~Hz}$, $1.5 \mathrm{~V}$ for $25 \mathrm{~min}$ ). GUVs were gently washed with HEPES buffer (25 mM HEPES pH7.4, $150 \mathrm{mM} \mathrm{NaCl}, 310 \mathrm{mOsmol} / \mathrm{kg}$ ) and collected via centrifugation (1200x g; $\left.25^{\circ} \mathrm{C} ; 5 \mathrm{~min}\right)$. The loose GUV pellet was carefully resuspended in a small volume of HEPES buffer and diluted again in $11.5 \mathrm{ml}$ buffer followed by centrifugation $\left(1200 \mathrm{x} \mathrm{g} ; 25^{\circ} \mathrm{C} ; 5 \mathrm{~min}\right)$. The supernatant was removed while the loose GUV pellet was carefully resuspended. Imaging chambers (LabTek) were incubated sequentially with $0.1 \mathrm{mg} / \mathrm{ml}$ Biotin-BSA (Sigma) and $0.1 \mathrm{mg} / \mathrm{ml}$ Neutravidin (Thermo Fisher Scientific) dissolved in MiliQ water.

\section{Microscope setup}

For the measurements, two microscope setups have been used: Setup 1 (Figure 2 in the manuscript) and Setup 2 (Figure 3 in the manuscript). 
Setup 1. This home built confocal microscope system consists of an Olympus IX71 inverted microscope body (Olympus, Hamburg, Germany) equipped with a 3D piezo positioner from Physik Instrumente (P-562.3CD stage controlled via E-710.3CD controller). The output of three picosecond pulsed diode laser heads PicoTA-532, LDH-P-C-470 and LDH-P-635 controlled via PDL 828 Sepia II laser driver (all devices from PicoQuant, Berlin, Germany) is coupled to a single polarization maintaining single mode fiber and collimated by an air space objective (UPlanSApo 4x, NA 0.16). The beam enters the frame via the rear port and is reflected towards the objective (UPlanSApo 60x w, NA 1.2) using a Chroma ZT375/473/532/635rpc quad-band dichroic mirror. The collected signal leaves the microscope frame through the left-hand side port (thus focused by the original tube lens) and passes through a 50 micrometer (diameter) pinhole at the focal plane. The beam is collimated and split between two SPAD detectors (\$PD-50-CTC, MicroPhotonDevices, Bolzano, Italy). For dual-color FCS measurement T635lpxr splitter and HQ515/50 and HQ697/58 filters were used. To identify leaky and nonleaky vesicles, HQ515/50 filter was replaced by HQ590/50. The filters are mounted in front of each detector. Time-tagged time-resolved single photon counting data acquisition was performed by HydraHarp400 Multichannel Picosecond Event Timer \& TCSPC Module controlled via SymPhoTime software (both from PicoQuant, Berlin, Germany).

Setup 2 consists of a modified commercial Olympus FluoView 1000 system equipped with a dual channel PicoQuant LSM Upgrade Kit. To image individual GUVs for their leakage state three color $(\mathrm{B} / \mathrm{G} / \mathrm{R})$ imaging FluoView software utilizing built-in photomultiplier tubes, beam-splitters and band-pass or long-pass filters selected from built-in wheels was used. After positioning the laser beam at the place of interest, the internal beam splitters of FV1000 were removed enabling the light to leave the scan-head via a 50-micron (diameter) multi-mode fiber leading into two pieces of tau-SPAD detectors, with a configurable beam splitting unit and dedicated filter holders in front of each detector. The filters and beam-splitters used for dual-color FCS were the same as in Setup 1. Time-tagged time-resolved single photon counting data acquisition was performed by a HydraHarp400 Multichannel Picosecond Event Timer \& TCSPC module, controlled via SymPhoTime64 software, which incorporates the control of PDL 828 Sepia II driver (PicoQuant, Berlin, Germany). The excitation system is home-built and consists of LDH-D-C-470, LDH-D-C640 diode laser heads and $543 \mathrm{~nm}$ HeNe continuum wave lasers. 
The excitation was performed in the pulse-interleaved excitation (PIE) mode, avoiding artifacts caused by spectral bleed-through. The repetition rate of each laser was set to $10 \mathrm{MHz}$, giving rise to a $100 \mathrm{~ns}$ long time span available for each fluorescence decay. The laser intensity at the back aperture of the objective was kept below $10 \mu \mathrm{W}$.

Determination of the readout parameters by dual $(+1)-F C S$

A GUV was selected and classified as a leaky or nonleaky one by means of AlexaFluor-532 (excitation $532 \mathrm{~nm}$, emission 590/50 nm). Leaky GUVs are defined as GUVs filled by Alexa-Fluor-532 by more than 20\%; similarly, nonleaky GUVs must be filled by less than $20 \%$. In the next step, a membrane was placed into the waist of 470 and $635 \mathrm{~nm}$ lasers and a set of 60 second long dual-color FCS measurements using 515/50 nm (FGF2-GFP) and 697/58 nm (DOPE-Atto-633) emission channels was performed. The obtained autocorrelation $(\mathrm{AC})$ curves were fitted by a model assuming two-dimensional diffusion in the membrane (bound FGF2-GFP and DOPE-Atto-633), free three-dimensional diffusion in the solution (FGF2-GFP in the bulk) and transition of the dye to the triplet state: ${ }^{3}$

$$
G(\tau)=1+\left(\frac{1}{N_{1+\left(\frac{\tau}{\mathrm{D}_{\mathrm{D}}}\right)}}+\frac{1}{N_{\text {free }}} \frac{1}{1+\left(\frac{\tau}{\tau_{\mathrm{D}, \text { free }}}\right) \sqrt{1+\mathrm{SP} \frac{\tau}{\mathrm{T}_{\mathrm{D}} \text { free }}}}\right) \frac{1-T+T \exp \left(-\frac{\tau}{\tau \mathrm{T}}\right)}{1-T} .
$$

Here $\tau$ is a so-called lag-time, $N$ and $N_{\text {free }}$ are the number of membrane-bound and free dye molecules in the confocal volume, $\tau_{\mathrm{D}}$ and $\tau_{\mathrm{D} \text {,free }}$ the diffusion times of membrane bound and membrane free dye, SP the structure parameter, $T$ the fraction of the dye in the triplet state and $\tau_{\mathrm{T}}$ the lifetime of the triplet state. In the beam center, the fluorescent signal coming from the solution is negligible. This simplifies the above equation to

$$
G(\tau)=1+\frac{1}{N} \frac{1}{1+\left(\frac{\tau}{\tau_{\mathrm{D}}}\right)} \frac{1-T+T \exp \left(-\frac{\tau}{\tau_{\mathrm{T}}}\right)}{1-T} .
$$

For this method, it is essential to determine all output parameters under conditions where the membrane is precisely focused into the beam center. To achieve this, a membrane was placed into the waist, moved about $1 \mu \mathrm{m}$ above the waist and scanned vertically along the $z$ axis in 10 to 15 steps spaced $150 \mathrm{~nm}$ from each other. Dual-color FCS measurements were performed at each step. The correct position of the membrane was indicated by the 
minimum in $N$ and $\tau_{\mathrm{D}}{ }^{4}$ These minimum values and the corresponding average intensity in counts per second $\langle I\rangle$ were taken for further analysis. Alternatively (data collected for Figure

3), a membrane was carefully placed into the beam center by finding the maximum in the emission $(697 / 58 \mathrm{~nm})$ along the vertical z-axis passing through the membrane, and a 60 second long dual-color FCS measurement was performed at that point.

The determination of the average oligomeric state on a single GUV is based on comparison of the brightness of an oligomer $\phi$ (oligo) to that of a monomer $\phi$ (mono). The

brightness of an oligomer is calculated as $\phi$ (oligo) $=\frac{(l)}{N}$. The brightness of a monomer $\phi$ (mono) is obtained in a similar manner as $\phi$ (oligo), but it needs to be ensured that the probability that two labelled FGF2-GFP molecules meet in a cluster is negligible. This was achieved by using His-FGF2-Y81pCMF-C77/95A-GFP which binds to DGS-NTA containing bilayer as a dimer at maximum and by diluting His-FGF2-Y81pCMF-C77/95AGFP by the unlabelled variant His-FGF2-Y81pCMF-C77/95A-GFP at the ratio 1:10. ${ }^{56}$ Finally, the average oligomeric state was calculated as

$$
N(\mathrm{~m} . \mathrm{u} .)=\frac{\phi \text { (oligo })}{\phi(\text { mono })} \text {. }
$$

The diffusion coefficient $D$ of FGF2-GFP and DOPE-Atto-633 was determined by using the following relationship

$$
D=\frac{\omega^{2}}{4 \tau_{\mathrm{D}}},
$$

where $\omega$ is the radius of the beam waist obtained by a standard calibration procedure. Good quality of the membrane was indicated by free diffusion of DOPE-Atto-633 in the bilayer and a reasonable diffusion coefficient obtained for this lipid.

Finally, the protein surface concentration was calculated as the number of protein molecules in the confocal spot $N($ oligo/mono $) * N($ m.u. $)$ of a known radius $\omega$ :

$$
c_{\text {surf }}=\frac{N(\mathrm{~m}, \mathrm{u}) N \text { (oligo/mono) }}{\pi \omega^{2}} .
$$

\section{Supporting Results}

Functional correlation of the diffusion coefficient with membrane pore formation 


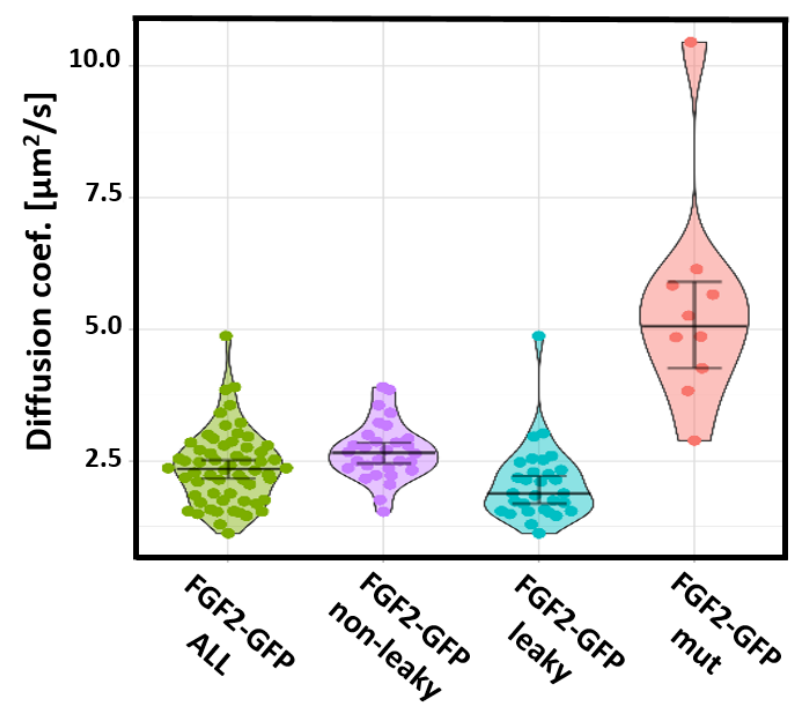

Figure SI 1: Functional correlation of the diffusion coefficent with membrane pore

formation. Each dot corresponds to a data-point measured on single GUV. In the first step, all GUVs were taken for the analysis (FGF2-GFP ALL). In the next step, the GUVs were sorted out depending on membrane permeability into nonleaky (FGF2-GFP nonleaky) and leaky (FGF2-GFP leaky) ones. For comparison, the plots for double cysteine mutant His-FGF2C77/95A-GFP where oligomerization is blocked are also shown (FGF2-GFP mut). Median values as well as $95 \%$ confidence intervals are indicated by the black solid lines.

\section{References}

(1) Young, T. S.; Ahmad, I.; Yin, J. A.; Schultz, P. G. An Enhanced System for Unnatural Amino Acid Mutagenesis in E. Coli. J. Mol. Biol. 2010, 395 (2), 361-374.

(2) García-Sáez, A. J.; Ries, J.; Orzáez, M.; Pérez-Payà, E.; Schwille, P. Membrane Promotes TBID Interaction with BCL XL. Nat. Struct. Mol. Biol. 2009, 16 (11), 1178-1185.

(3) Widengren, J.; Mets, Ü.; Rigler, R. Fluorescence Correlation Spectroscopy of Triplet States in Solution: A Theoretical and Experimental Study. J.Phys.Chem. 1995, 99, 13368-13379.

(4) Benda, A.; Beneš, M.; Mareček, V.; Lhotský, A.; Hermens, W. T.; Hof, M. How to Determine Diffusion Coefficients in Planar Phospholipid Systems by Confocal Fluorescence Correlation Spectroscopy. Langmuir 2003, 19 (10), 4120-4126.

(5) Steringer, J. P.; Lange, S.; Čujová, S.; Šachl, R.; Poojari, C.; Lolicato, F.; Beutel, O.; Müller, H. M.; Unger, S.; Coskun, Ü.; et al. Key Steps in Unconventional Secretion of Fibroblast Growth Factor 2 Reconstituted with Purified Components. Elife 2017, 6, 1-36.

(6) Müller, H. M.; Steringer, J. P.; Wegehingel, S.; Bleicken, S.; Münster, M.; Dimou, E.; Unger, S.; Weidmann, G.; Andreas, H.; García-Sáez, A. J.; et al. Formation of Disulfide Bridges Drives Oligomerization, Membrane Pore Formation, and Translocation of Fibroblast Growth Factor 2 to Cell Surfaces. J. Biol. Chem. 2015, 290 (14), 8925-8937. 